\title{
Electrophysiological features of central motor conduction in spinocerebellar atrophy type 1 , type 2, and Machado-Joseph disease
}

\author{
T Yokota, H Sasaki, K Iwabuchi, T Shiojiri, A Yoshino, A Otagiri, A Inaba, T Yuasa
}

\begin{abstract}
Objectives-To characterise electrophysiologically the central motor conduction of spinocerebellar atrophy type 1 (SCA1), type 2 (SCA2), and Machado-Joseph disease (MJD).

Methods-Motor evoked potentials (MEPs) triggered by transcranial magnetic stimulation (TMS) was used to investigate the functions of corticospinal tracts of 10 patients with SCA1, 10 with MJD, and eight with SCA2 in addition to pathological study of the spinal cord in a patient with SCA1.

Results-Central motor conduction time (CMCT) was extremely prolonged and the MEP threshold increased in all patients with SCA1, whereas both were normal in patients with SCA2 or MJD. The MEP size in MJD was larger than normal, but was normal in SCA1 and SCA2. A pathological investigation of the corticospinal tract of the spinal cord of a patient with SCA1 showed selective loss of large diameter fibres.
\end{abstract}

Department of

Neurology, Tokyo

Medical and Dental

University, Tokyo,

Japan

T Yokota

H Shiojiri

A Yoshino

A Otagiri

A Inaba

T Yuasa

Department of

Neurology, Hokkaido

University School of

Medicine, Hokkaido,

Japan

H Sasaki

Department of Neurology and Psychiatry, Kanagawa

Rehabilitation Center, Kanagawa, Japan

K Iwabuchi

Correspondence to:

Dr Takanori Yokota,

Program Aging, The

Burnham Institute, $10901 \mathrm{~N}$

Torrey Pines Road, La Jolla,

CA 92037, USA. Telephone

+16196463100 ext 3724;

fax +1 619646 3192; email

fyokota@lgcrf.edu

Received 8 August 1997 and in final revised form 24

February 1998

Accepted 25 February 1998 volvement of the corticospinal tract in either disease. ${ }^{1-4}$ We investigated the function of the

Table 1 Patient profile

$\mathrm{AH}=$ abductor hallucis muscle. central motor tract using the motor evoked potential (MEP) triggered by transcranial magnetic stimulation. The pathophysiology of the pyramidal tract lesions in the diseases is discussed.

\section{Patients and methods}

Ten patients with SCA1, 10 with MJD, eight with SCA2, and 16 age matched controls were studied. The pyramidal features and muscle strengths are presented in table 1 . CAG expansions at each chromosome were used for the diagnosis of SCA $1,{ }^{5}$ SCA $2,{ }^{6}$ and MJD. ${ }^{7}$

After giving their informed consent, the patients and controls underwent transcranial magnetic stimulation (Magstim 200, Magstim Company Ltd, UK). The round coil $(14 \mathrm{~cm}$ diameter, 2.0 Tesla) was centred over the vertex to stimulate the hand muscle and was placed with the lower edge on the vertex to stimulate the foot muscle. Anticlockwise oriented current was used to stimulate the left hemisphere, and recordings were made from the first dorsal interosseous and abductor hallucis muscles via surface electrodes attached in a belly-tendon montage. To record the cortical response, subjects were asked to contract the target muscles with about $50 \%$ of the maximum muscle force, which was monitored with a force transducer. To cancel the volume conducted potential of the hand muscles innervated by the median nerve, the median nerve was stimulated at the wrist when the cortex was stimulated. More than eight responses to cortical stimulation were recorded at the maximum output of the stimulator, and the shortest latency, largest amplitude, and the area of MEP were measured. For evaluation of peripheral motor conduction time (PMCT), magnetic stimulation was applied over the C7 and the L5 vertebrae with a figure of eight coil $(9 \mathrm{~cm}$ diameter, 2.2 Tesla) with maximum output (PMCTspine). In addition, PMCT was also calculated from the latency of the $F$ wave (PMCTF). Subtraction of PMCTspine and

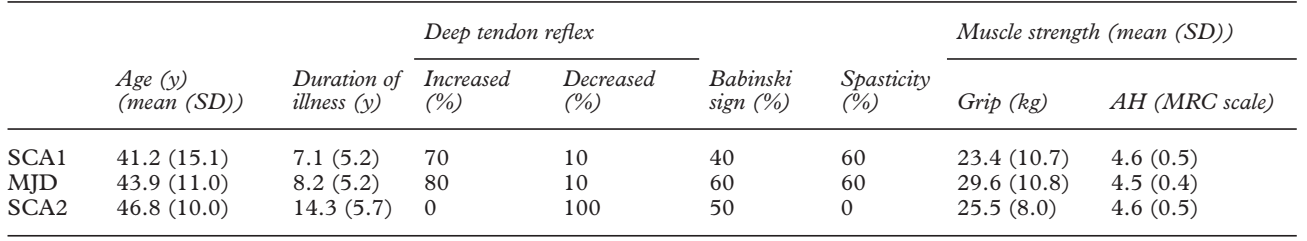


Table 2 Results of motor evoked potential (MEP) study

\begin{tabular}{|c|c|c|c|c|c|c|c|c|c|}
\hline & \multicolumn{6}{|l|}{$F D I$} & \multicolumn{3}{|l|}{$A H$} \\
\hline & $\begin{array}{l}\text { CMCT spine } \\
\text { (ms) }\end{array}$ & $\begin{array}{l}C M C T F \\
\text { (ms) }\end{array}$ & $M E P / M(a m p)$ & $M E P / M$ (area) & MEP Th (\%) & $\begin{array}{l}M D / M E P \\
\text { (area) }\end{array}$ & $\begin{array}{l}\text { CMCT spine } \\
\text { (ms) }\end{array}$ & CMCTF (ms) & $M E P / M(a m p)$ \\
\hline SCA1 & $10.7(1.9)^{\star}$ & $9.5(1.6)^{\star}$ & $0.37(0.13)$ & $0.83(0.28)$ & $64.3(21.1)^{\star}$ & $0.30(0.22)$ & $24.7(2.7)^{\star}$ & $21.3(3.5)^{\star}$ & $0.16(0.08)$ \\
\hline MJD & $6.3(0.5)$ & $4.5(0.8)$ & $0.70(0.19)^{\star}$ & $1.24(0.37)^{\star}$ & $40.0(10.0)$ & $0.63(0.12)^{\star}$ & $16.0(1.0)$ & $13.2(0.9)$ & $0.35(0.15)^{\star}$ \\
\hline SCA2 & $6.5(0.4)$ & $5.1(0.4)$ & $0.48(0.11)$ & $0.79(0.10)$ & $44.2(3.9)$ & $0.42(0.27)$ & $15.5(1.9)$ & $13.1(1.6)$ & $0.17(0.13)$ \\
\hline Normal & $6.1(0.7)$ & $4.8(1.1)$ & $0.39(0.13)$ & $0.80(0.16)$ & $41.2(3.8)$ & $0.35(0.16)$ & $15.1(1.2)$ & $13.0(1.7)$ & $0.14(0.11)$ \\
\hline
\end{tabular}

${ }^{\star} \mathrm{p}<0.01$.

Values are mean (SD).

$\mathrm{MD}=$ multiple discharge; $\mathrm{Th}=$ threshold .

PMCTF from MEP latency gave central motor conduction time (CMCTspine) and CMCTf, respectively. ${ }^{8}$ MEP size was evaluated as the ratio to the size of the $M$ wave of the peripheral stimulation at the wrist and ankle in peak to peak amplitude and positive and negative areas (MEP/M amplitude and area ratios). To record the MEP of the first dorsal interosseous due to multiple discharges of the $\alpha$-motor neuron produced by single cortical stimulation (MD), the ulnar as well as the median nerve was supramaximally stimulated at the time of cortical stimulation for the collision of first descending volleys at the peripheral nerve. ${ }^{9}$ The MEP threshold stimulation intensity was defined as the stimulus intensity required to produce an MEP of at least $0.1 \mathrm{mV}$ amplitude in three of five trials.

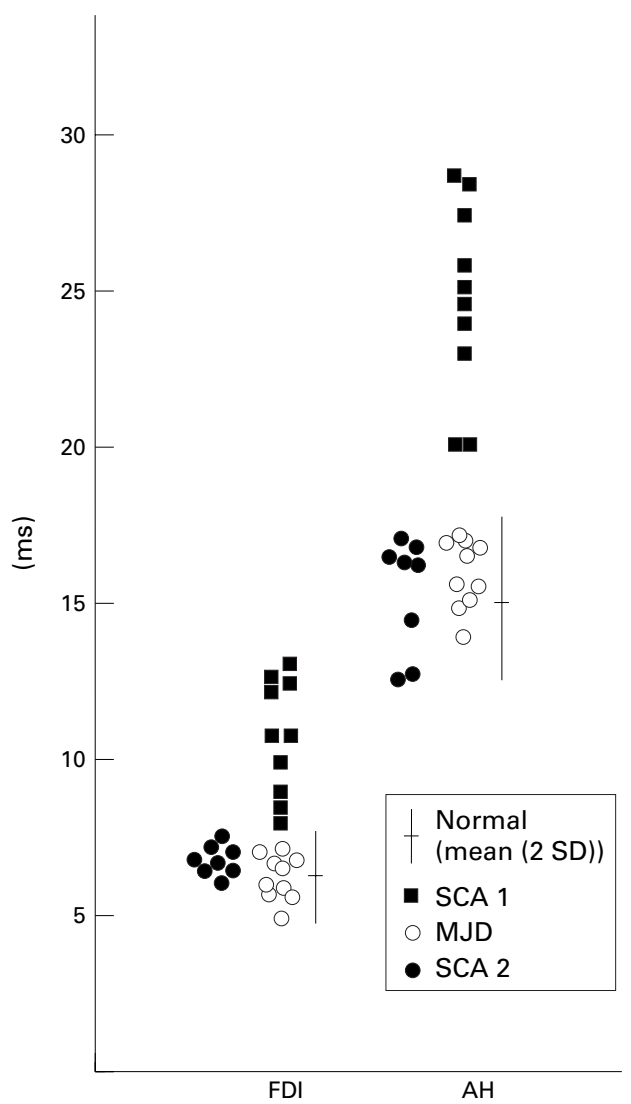

Figure 1 CMCTspine in SCA1, SCA2, and MFD, The CMCTs of both the first dorsal interosseous (FDI) and abductor hallucis $(A H)$ were markedly prolonged in almost all the patients with SCA1, but were normal in all the patients with MFD and patients with SCA2. There was no overlap of CMCTs of abductor hallucis between patients with SCA1 and those with MFD or SCA2.
The non-parametric Mann-Whitney $U$ test was used to analyse the MEP data of the patients and normal subjects and correlations with CAG repeat numbers for clinical and electrophysiological results.

A neuropathological examination of the spinal cord at th 5 was carried out at postmortem in a patient with SCA1. The sections were fixed in $1 \%$ osmium tetroxide and stained with haematoxylin and eosin, Sudan black B, KlüverBarrera, Holzer, and Bodian stains. This patient was the older sister of one of our patients with SCA1 who was electrophysiologically examined. These sisters had the same age of onset (41 years), number of CAG repeats (49), and duration of illness (13 years). The necropsied patient had not had an electrophysiological examination, but the CMCTspine and CMCTf of the abductor hallucis in her younger sister were markedly prolonged (26.4 and $23.8 \mathrm{~ms}$ ). The pyramidal features of both patients were similar (positive Babinski's sign, no spasticity, normal tendon reflexes, and mild muscle weakness). Detailed clinical data on these siblings have been reported elsewhere. ${ }^{10}$

\section{Results}

Results of the electrophysiological study are given in table 2. CMCTspine and CMCTf of both the first dorsal interosseous and abductor hallucis were markedly prolonged in almost all the patients with SCA $1(\mathrm{p}<0.01)$, whereas they were normal in all the patients with MJD or SCA2 (figs 1, 2). There was no overlap of CMCTs of the abductor hallucis between SCA1 and MJD or SCA2 (fig 1). The threshold stimulation intensity for MEP was increased in all patients with SCA1 $(p<0.01)$, and normal in patients with MJD or SCA2. Both the MEP/M amplitude and area ratios were increased in the patients with MJD $(\mathrm{p}<0.01$, fig 2), but were normal in the patients with SCA and SCA2. The multiple discharge of $\alpha$-motor neurons excited by cortical stimulation was also significantly larger in patients with MJD, but was normal in patients with SCA1 (fig 3).

Age of onset was inversely correlated with the CAG repeat number of mutant allele in all groups $(p<0.01)$. There was no significant correlation for any disease for the CAG repeat number, electrophysiological findings (CMCT, MEP/M ratios, MEP threshold), pyramidal tract symptoms (tendon reflexes, spasticity, Babinski's sign, and muscle strengths), and the duration of illness. 

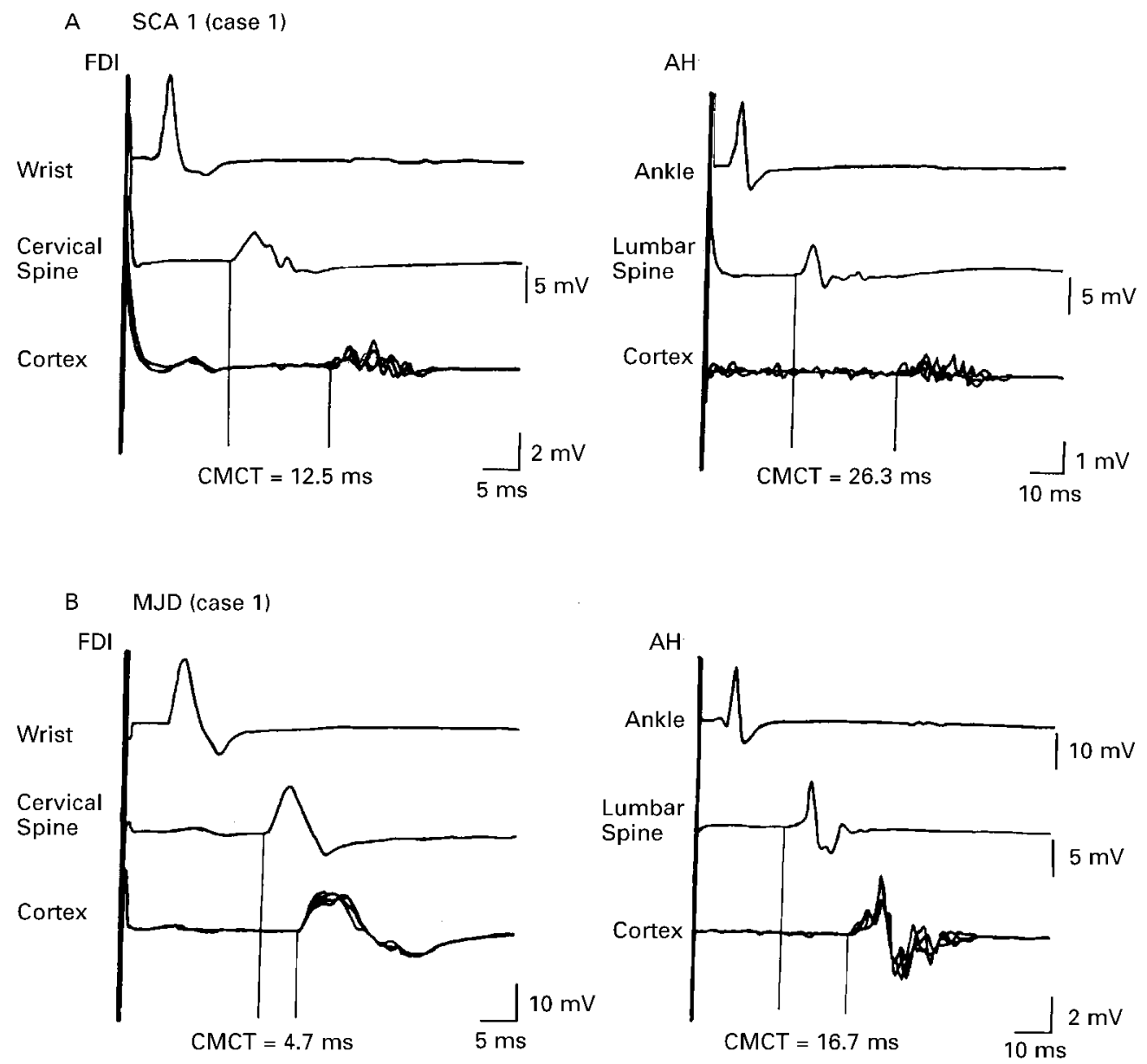

Figure 2 (A) The wave forms of MEPs of a patient with SCA1 and (B) a patient with MFD. The CMCT was markedly prolonged in the patient with SCA1, and was normal in the patient with MFD. The MEP was large in the patient with MFD and was dispersed in the patient with SCA1.

A study of the pathology of the thoracic cord of a patient with SCA1 showed selective loss or axonal atrophy of large myelinated fibres (diameter $>10 \mu \mathrm{m}$ ) but most smaller fibres were preserved as shown by Sudan black B for myelin sheaths (fig 4). There was a slight fibrillary gliosis, but the fibre loss could not be detected by other routine stains.

\section{Discussion}

Prolonged CMCT is caused by the following mechanisms: (1) conduction delay in the fast pyramidal tract axon due to demyelination or axonal atrophy; (2) activation of the $\alpha$-motor neuron via slow pyrimidal tract axons or polysynaptic descending pathways; and (3) prolonged rising time of the membrane potential of the $\alpha$-motor neuron in reaching the

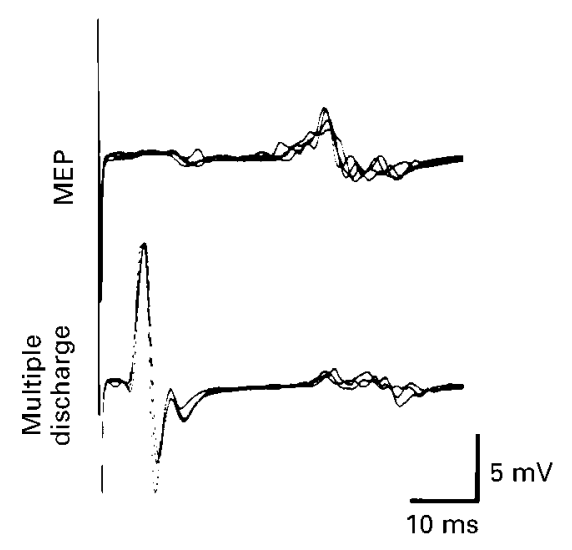

SCA1 (case 2)

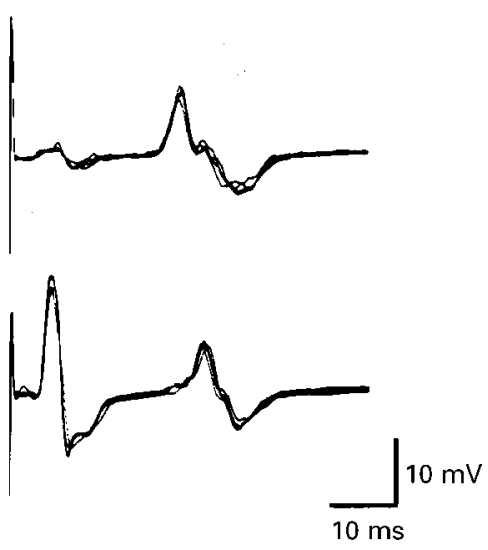

MJD (case 8)

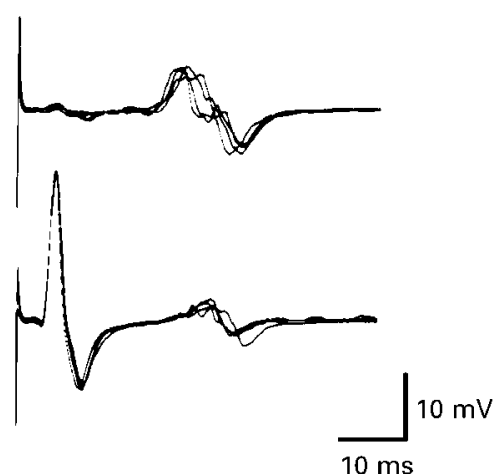

Normal

Figure 3 MEP by multiple discharge of a-motor neuron. The MEPs not collided by peripheral nerve stimulation were larger than normal in patient 8 with $M F D$. 

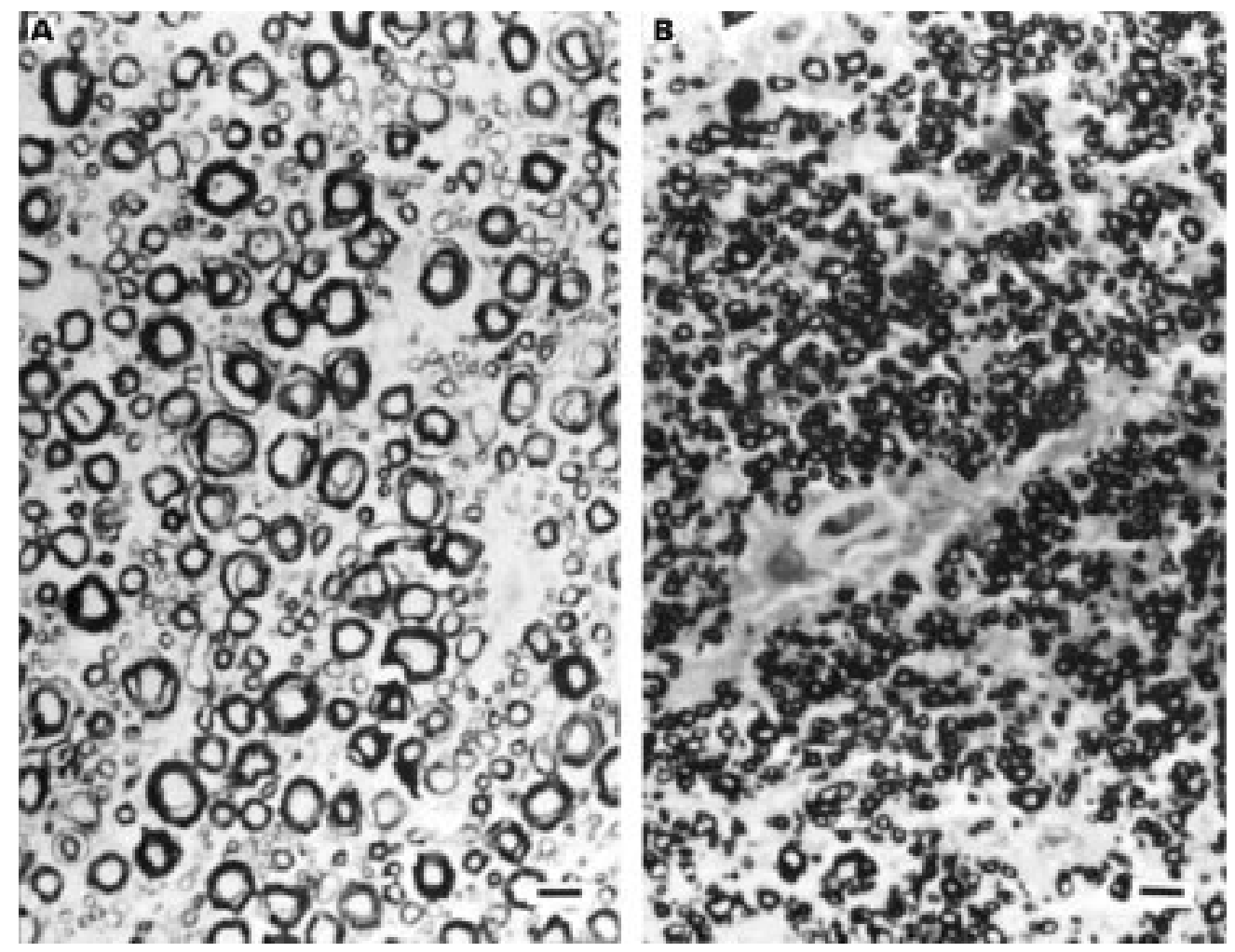

Figure 4 Transverse sections of the lateral corticospinal tract of the spinal cord at th5 in a patient with SCA1 (right) and a control (left). Sudan black B staining. Bar=10 $\mu \mathrm{m}$. The density of large myelinated fibres in the patient with SCA1 is markedly decreased.

threshold for the action potential due to decreased excitability of the $\alpha$-motor neuron or the decreased size of the EPSPs from the pyrimidal tract. In SCA1, CMCT was prolonged, but the MEP size and $\mathrm{MD} / \mathrm{MEP}$ ratio were in the normal range. These findings indicate that the conduction of the pyrimidal tract is slowed, but that its connection is preserved. This interpretation is supported by the fact that muscle weakness was minimal in these patients. The pathology in the SCA1 necropsy showed selective large fibre loss or axonal atrophy in the corticospinal tract. Large fibres with diameters of more than $10 \mu \mathrm{m}$ are considered to arise from the Betz cells in the primary motor cortex and to end directly on the $\alpha$-motor neuron. ${ }^{11}$

Demyelination and axonal atrophy is improbable because thin or thick myelin, or a redundancy of myelin, was rarely seen. We hypothesise that the fast and large pyrimidal tract axons are selectively degenerated and that the small and slow pyramidal tract axons, or the polysynaptic pathway, activates the $\alpha$-motor neuron in SCA1. However, the pathological findings of one patient alone cannot rule out the possibility of conduction slowing of the fast pyramidal tract axons.

The MEP threshold stimulation intensity was also abnormal only in SCA1, although the responsible lesion site could not be determined. Increased intensity for MEP threshold has been known to be caused by dysfunction of the pyrimidal tract axons or interneurons in the primary motor cortex, but could be influenced by changes in tuning of the primary motor cortex from the basal ganglia, cerebellum, or other cortex.

Interestingly, we found the MEP size to be abnormally large in MJD, the MEP area recorded from first dorsal interosseous being larger than the $M$ wave area. This is caused in part by increased multiple discharges of the $\alpha$-motor neuron by single cortical stimulation. Whether this hyperexcitability of the central motor tract in MJD occurs at a cortical, or spinal level, or both, could not be determined. We speculate, however, that it is caused at the cortical level because this MEP abnormality was not correlated with increased tendon reflex.

Studies of genotype and neurological symptoms have shown that the number of CAG repeats correlates with the severity of cerebellar deficits in SCA1, ${ }^{12}$ and with the severity of ataxia, abnormal tendon reflexes, and decreased vibration sense in MJD. ${ }^{4}$ In our study the electrophysiological abnormalities of the central motor tract were not clearly correlated with the CAG repeat number. A definitive conclusion awaits further studies of a large number of patients, because age and duration of illness at the time of examination seem to affect the correlations, although not significantly. No correlation was found between the abnormal results in the MEP study and pyramidal tract symptoms (tendon reflex abnormality, Babinski's sign, spasticity). This indicates that the pyramidal tract symptoms seen in these diseases are not caused by dysfunction of the 
strictly defined pyramidal tract axon-that is, from Betz cells to $\alpha$-motor neurons.

In summary, our results clearly show that there is a marked difference in the pathophysiology of the central motor tract in SCA1, MJD, and SCA2; prolonged CMCT and increased MEP threshold in SCA1, increased MEP size in MJD, and no abnormality in SCA2. The prolonged CMCT for the lower limb muscle in SCA1 is so significant that it can be used to differentiate SCA1 from MJD or SCA2, although the clinical manifestations of dominant inherited ataxias are sometimes very similar. ${ }^{13} 14$

We are grateful to Drs Tatsuro Oda and Fumiko Hirashima for their help.

\section{Addendum}

During the revision process of this article, a similar study on MEP in SCA 1-3 patients has been published (Brain 1998;120:2141-8).

1 Iwabuchi $\mathrm{K}$, Ikeda $\mathrm{T}$, Takahata $\mathrm{N}$, et al. A clinicopathological study on spinocerebellar ataxia 1 (SCA1) - special references to the findings of the family reported by Yakura erences to the findings of the family reported by
(1974). Adv Neurol Sci (Tokyo) 1992;36:665-78.

2 Sequeiros J, Coutinho P. Epidemiological and clinical aspects of Machado-Joseph disease. In: Harding $\mathrm{AE}$ et al, eds. Inherited ataxias: advance in neurology. Vol 61. New York: Raven Press, 1993:139-53.
3 Tsuchiya K, Wakabayashi M, Oyanagi S, et al. MachadoJoseph disease in Japan: clinicopathological study of 6 autopsy cases with special reference to the clinicopathological correlation to cerebellar ataxia and lower motor neuron signs. Neuropathology 1994;14:13-36.

4 Durr A, Stevanin G, Cancel G, et al. Spinocereballar ataxia 3 and Machado-Joseph disease: clinical, molecular, and neuropathological features. Ann Neurol 1996;39:490-9.

5 Orr HT, Chung M, Banfi S, et al. Expansion of an unstable trinucleotide CAG repeat in spinocerebellar ataxia type 1. Nat Genet 1993;4:221-6.

6 Sanpei K, Takano H, Igarashi S, et al. Identification of the gene for spinocerebellar ataxia type 2 (SCA2) using a direct dentification of repeat expansion and cloning technique (DIRECT). Nat Genet 1996;14:277-83.

7 Kawaguchi Y, Okamoto T, Taniwaki $M$, et al. CAG expansions in a novel gene for Machado-Joseph disease at chromosome 14q32.1. Nat Genet 1994;8:221-8.

8 Eisen A, Shybel W. Clinical experience with transcranial magnetic stimulation. Muscle Nerve 1990;13:995-1011.

9 Day BL, Rothwell JC, Thompson PD, et al. Motor cortex stimulation in intact man: 2 . Multiple descending volleys. Brain 1987;110:1191-209.

10 Hanihara $\mathrm{T}$, Takahashi $\mathrm{T}$, Inoue $\mathrm{K}$, et al. Sibilings with spinocerebellar ataxia type 1 (SCA1) - diagnosis by detecting the expansion of CAG repeat on chromosome 6q. Clin Neurol (Tokyo) 1994:34:508-10.

11 Jones EG, Wise SP. Size, laminar and columnar distribution of efferent cells in the sensori-motor cortex of the monkeys. f Comp Neurol 1977;175:391-438.

12 Goldfarb LG, Vasconcelos O, Platonov FA, et al. Unstable triplet repeat and phenotypic variability of spinocerebellar ataxia type 1. Ann Neurol 1996;39:500-6.

13 Dubourg O, Durr A, Cancel G, et al. Analysis of the SCA1 CAG repeat in a large number of families with dominant ataxia: clinical and molecular correlations. Ann Neurol 1995;37:176-80.

14 Sasaki H, Fukazawa T, Yanagihara T, et al. Clinical features and natural history of spinocerebellar ataxia type 1. Acta Neurol Scand 1996;93:64-71. 